\title{
La dynamis des anneaux en Grèce antique
}

\author{
Charles Delattre \\ Université de Paris Ouest Nanterre, THEMAM (UMR 7041 ArScAn) \\ charles.delattre@u-paris10.fr
}

Recibido: $16-04-2012$

Aceptado: 20-12-2012

\section{RÉSUMÉ}

Dans les récits grecs comme dans ceux d'aujourd'hui, certains objets semblent posséder un pouvoir magique, une force interne que l'heureux propriétaire de l'objet peut utiliser à son profit. À l'inverse, certains objets, pourtant identiques, en semblent privés. À partir d'une étude centrée sur les anneaux, nous proposons d'unifier ces deux catégories et de redéfinir la notion de 'pouvoir magique', non comme une variation du mana défini par M. Mauss, mais comme la partie prenante d'un conflit entre différentes forces à l'œuvre. La magie apparaît dès lors non comme une catégorie à part, mais comme l'une des composantes d'un monde socialement traversé par des conflits.

Mots clé: Anneau, mythe, magie, dynamis, pouvoir, cycle.

\section{RESUMEN}

En los cuentos de la Grecia antigua como en los de hoy, ciertos objetos parecen poseer un poder mágico, una fuerza interna que el propietario del objeto puede utilizar en su provecho. Por el contrario, otros objetos, sin embargo idénticos, parecen carecer de tal poder. A partir de un estudio centrado sobre los anillos proponemos unificar estas dos categorías y volver a definir la noción de 'poder mágico': el poder mágico no es un avatar del mana definido por M. Mauss, sino un elemento que participa en un conflicto entre diferentes fuerzas. La magia aparece desde ese momento no como una categoría separada, sino como uno de los componentes de un mundo socialmente cruzado por conflictos.

Palabras clave: anillo, mito, magia, dynamis, poder, ciclo

\begin{abstract}
In Greek and modern tales, some objects seem to possess a magic power, an internal strength that the owner of the object can use. On the contrary, certain objects, nevertheless identical, seem to lack suche a power. Within the frame of a study centered on rings, we suggest unifying these two categories and redefining the notion of 'magic power' : 'magic power' is not an avatar of the mana defined by M. Mauss, but is the member of a conflict between various strengths at work. Magic appears from then on not as a category per se, but as one of the components of a world socially ridden with conflict.
\end{abstract}

Keywords: ring, myth, magic, dynamis, power, cycle. 


\section{DÉFINITION GÉNÉRALE : UN SYSTÈME DUEL ?}

Définir un anneau magique ne pose pas à priori de problème : même sans avoir lu le Seigneur des anneaux de Tolkien, ou sans avoir vu les versions cinématographiques de P. Jackson, la plupart des occidentaux savent bien que certaines bagues sont dangereuses à porter, parce qu'elles recèlent de mystérieux pouvoirs que leur possesseur ne saura pas toujours maîtriser ${ }^{1}$. Mais il existe en fait deux types d'anneaux dans les récits et, plus largement, dans la documentation textuelle héritée du monde grec antique : seul le premier peut être qualifié de 'magique', tandis que les propriétés du second semblent appartenir à un autre registre.

\subsection{L'anneau d'invisibilité}

Le premier type d'anneau est celui qui est capable de conférer le pouvoir d'invisibilité. On en trouve la première description dans un célèbre passage de la République, où Glaucon, l'un des frères de Platon, à l'occasion d'un débat sur la notion de justice, rapporte à titre d'exemple l'histoire de Gygès, ancêtre fondateur de la dynastie royale de Lydie 2 . L'anecdote se décompose en termes simples : par un concours de circonstances, Gygès découvre un anneau ; toujours par hasard, il découvre que cet anneau

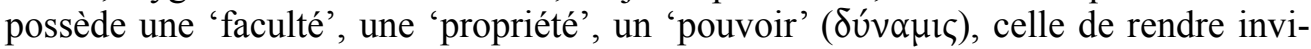
sible qui le porte. Gygès utilise alors l'anneau pour remplir trois objectifs, séduire la reine, tuer le roi et enfin s'emparer du 'pouvoir' (’’ $\rho x \eta ́)$. Pour finir, le nouveau statut de Gygès est défini par Glaucon comme celui de jouir de la 'liberté' ( $\varepsilon$ $\xi o v \sigma i ́ \alpha)$ de faire

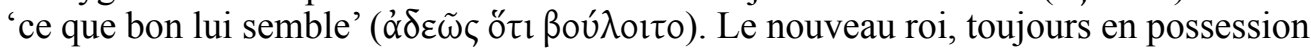
de son anneau magique, est même considéré comme 'équivalent à un dieu' (iøó $\theta \varepsilon \circ \varsigma$ ).

Des éléments similaires composent un récit d'Hérodote qui porte sur l'accession de Gygès au pouvoir ${ }^{3}$ : on y retrouve en particulier l'aide fournie par la reine à Gygès pour se défaire du roi en titre. Mais il faut immédiatement souligner une différence essentielle, qui réoriente l'interprétation du récit de Glaucon chez Platon : l'anneau

${ }^{1}$ Certaines parodies circulant sur Internet ne sont compréhensibles que dans ce contexte : une photo prise en 2004 montre par exemple l'ancien président des Etats-Unis, George W. Bush, portant ce qui semble être une alliance, et qu'un agrandissement identifie comme étant l'anneau du Seigneur du Mal. Une légende, «Frodo has failed, Bush has the ring», accentue la charge polémique de l'image (http://politicalhumor.about.com/library/images/blbushlordrings.htm). Une simple enquête sur moteur de recherche (mots-clés «Bush ring») montre la diversité des versions, posters, graffitis, voire autocollants pour automobile qui reprennent ce motif.

${ }^{2}$ Platon, République, II, 359b-360b. Gygès doit être identifié, malgré des divergences de détail, avec Guggu, roi des Luddi, qui entretint des liens de vassalité avec l'empire assyrien et qui combattit contre les Cimmériens ( $c f$. AsHERI \& ALII (2007) 83).

${ }^{3}$ Hérodote, I, 7, 1-14. Le récit d'Hérodote développe considérablement le contexte historique de l'anecdote de Gygès, même s'il existe des divergences par rapport à ce que l'on peut tirer des tablettes assyriennes où Guggu est nommé. L'histoire de Gygès met en scène en particulier le passage du pouvoir dynastique en Lydie de Myrsilos / Candaule aux Mermnades, dont Crésus est le dernier descendant. Pour d'autres versions sur Gygès, $c f$. Nicolas de Damas, FGrHist, IIa $90 \mathrm{~F} 47$ et les seize trimètres iambiques conservés par un papyrus du $\mathrm{II}^{\mathrm{e}}$ ou du $\mathrm{III}^{\mathrm{e}} \mathrm{s}$. de notre ère et identifiés comme un morceau de poésie dramatique (Tragica Adespota 664 Snell \& Kannicht). 
magique est totalement absent de la version hérodotéenne, où Gygès se rend invisible grâce à un simple subterfuge, qui consiste à se dissimuler derrière un rideau.

On peut imaginer que les deux versions sont indépendantes, ou que celle de Platon, qui comprend des éléments merveilleux, est en fait plus ancienne que celle d'Hérodote, qui se serait inspiré de la source qu'aurait également utilisée Platon ${ }^{4}$. Mais il me paraît plus vraisemblable, car plus en accord avec la manière de Platon, de considérer que le récit de la République est une réécriture ironique et consciente, et que l'introduction du motif de l'anneau magique sert un motif précis. Le thème du 'pouvoir magique' ( $\delta u ́ v \alpha \mu \iota \varsigma)$ de l'anneau ne doit en effet pas être considéré comme anodin chez Platon, car il entre en résonance avec le but poursuivi par Gygès, s'emparer du 'pou-

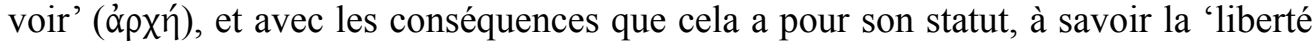

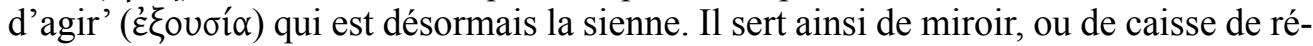
sonance, au motif plus général qui est celui du débat entre Glaucon et Socrate, concer-

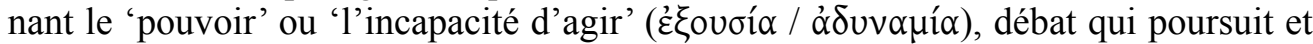
reformule celui auquel Socrate s'est livré avec Thrasymaque, sur la question de l'agir justement, du droit du plus fort et du profit que l'on peut avoir à être juste ou injuste.

Il n'y a d'ailleurs pas de mystère quant à la fonction du récit, car celui-ci développe explicitement une proposition de Glaucon :

«Donnons à l'homme de bien et au méchant un égal pouvoir de faire ce qui leur plaira; suivons-les ensuite et regardons où la passion va les conduire $\iota^{5}$.

Cette proposition ne définit pas une expérimentation en cognitive sciences, mais un cas fictif, caractérisé comme tel par Glaucon ${ }^{6}$, que le récit de Gygès complète. L'histoire accomplit exactement ce qui a été énoncé en introduction par Glaucon : elle sert d'emblème, plus encore que d'exemple, à une affirmation, et vaut donc démonstration de l'idée que la justice est seulement 'incapacité' ( $\alpha \delta v v \alpha \mu i ́ \alpha)$ à agir de manière injuste, une idée qui sera bien sûr mise en pièces par Socrate dans la suite du débat.

Quel que soit le rôle de l'anneau magique dans l'économie de l'énoncé de Platon, il se rattache sans difficulté à une catégorie générale, celle des objets magiques qui modifient la capacité à voir. Platon lui-même ${ }^{7}$ compare d'ailleurs 1'anneau de Gygès au casque d'invisibilité d'Hadès, la kuṽ̃, que plusieurs auteurs associent aux exploits de Persée face à la Gorgone ${ }^{8}$. Le manteau d'invisibilité de Harry Potter, dans les

\footnotetext{
${ }^{4}$ La source d'Hérodote ne peut en tout cas pas être Xanthos de Lydie (FGrHist, IIIb F 765), qui est légèrement postérieur.

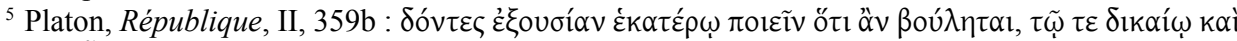

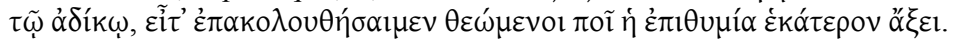

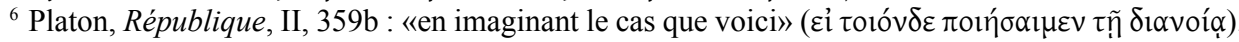

${ }^{7}$ Platon, République, X, 612a-b.

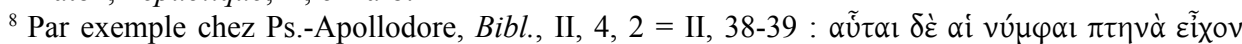

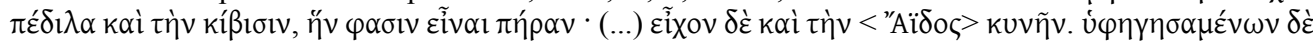

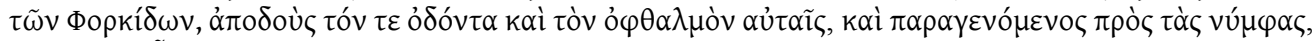

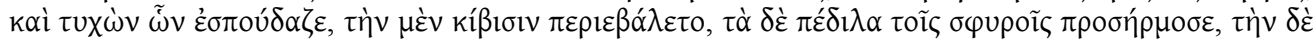

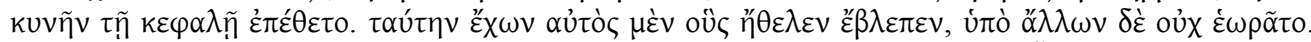

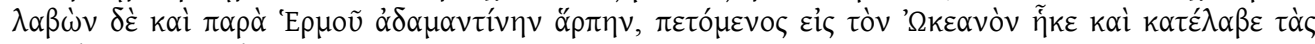

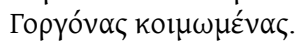


romans de J. K. Rowling, en est l'un des avatars modernes. Mais on peut aussi étendre la définition de cette catégorie d'objets dans un autre sens. En ne se bornant pas à ceux qui accordent le pouvoir d'invisibilité, on peut inclure les objets qui ont une action exactement inverse : certains anneaux modifient les capacités de vision en augmentant la clairvoyance du possesseur qui regarde, au lieu de diminuer celle de celui qui regarde le possesseur. C'est le cas, par exemple, dans le Roland furieux que l'Arioste a publié en 1532, de l'anneau donné par Mélisse à Roger pour se défaire des maléfices de l'enchanteresse Alcine : le héros est invité à voir de ses propres yeux, auxquels l'anneau a rendu leur pleine puissance, la laideur de celle qui a pensé le prendre au piège 9 .

Dans tous les cas, l'objet est dit 'magique', dans les langues modernes, parce qu'il

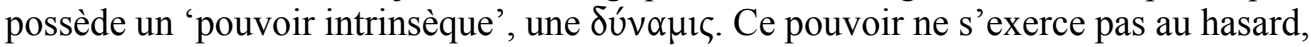
car il ne fonctionne que dans une relation étroite à celui qui porte l'anneau. Ce qui est une 'propriété' de l'objet concerne directement celui qui en est le 'propriétaire', même à titre temporaire. Le pouvoir magique de l'anneau d'invisibilité et de ses substituts n'est donc pas du type de celui qui peut être conféré, dans le cadre d'un rituel, à un objet qui en sera investi pour un laps de temps donné ${ }^{10}$ : il fait partie de la définition même de l'objet, en ceci qu'il ne dit pas seulement ses capacités d'intervention, mais aussi son statut de 'bien possédé' ( $\kappa \tau \tilde{\eta} \mu \alpha)$.

\subsection{L'anneau et son cycle}

Sans être doté de pouvoirs magiques, un deuxième type d'anneau se conduit dans les récits comme s'il avait malgré tout des facultés hors du commun. On ne peut cependant définir ces facultés autrement que par le rôle que l'anneau joue dans l'intrigue, non par les effets d'un pouvoir intrinsèque ${ }^{11}$. Sans pouvoir spécifique, l'anneau est d'une mobilité extrême, passe de main en main, et ses aventures ont une incidence directe sur le destin du personnage principal, son propriétaire le plus souvent ou l'un de ses proches.

On peut de ce fait définir l'intrigue non en fonction des personnages et de leurs actions, mais en se concentrant sur la trajectoire de l'anneau. Celui-ci quitte les mains de son propriétaire au début de l'histoire, et met ainsi en marche le moteur du récit ;

«Ces Nymphes avaient en leur possession des sandales ailées et la kibisis qui était, dit-on, une besace. (...) Elles avaient aussi le casque de cuir $<$ d'Hadès $>$. Les Phorcides lui ayant indiqué le chemin, il leur rendit leur dent et leur œil, alla trouver les Nymphes et obtint ce qu'il désirait : il mit sur lui la besace, ajusta à ses chevilles les sandales et mit le casque sur sa tête. Avec ce casque, il pouvait voir qui il voulait sans être vu d'autrui. Après avoir encore reçu d'Hermès une faucille d'acier, il arriva en volant à l'Océan et y trouva les Gorgones endormies» (trad. J.-Cl. Carrière \& B. Massonie).

${ }^{9}$ Arioste, Orlando furioso, VII, 70-74. Les Bijoux indiscrets de Diderot, publiés anonymement en 1748 , reprennent le thème de la clairvoyance en lui donnant une inflexion grivoise : l'anneau magique permet au sexe de la femme qui le porte d'acquérir la parole et de révéler ses secrets les plus intimes.

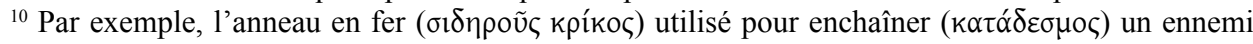
ou une femme (PGM V, 305-369) : on en dessine la circonférence sur un papyrus, que l'on surcharge de formules diverses, on coud l'anneau dans le papyrus même, en le plaquant sur le dessin qui le représente, et, après quelques prières, on jette le tout soit dans une tombe, soit dans une fontaine abandonnée.

${ }^{11}$ On trouvera plus de développements sur cette catégorie d'anneaux et sa définition dans DeLATTRE (2009) 
son absence sert de toile de fond aux agissements du propriétaire (colère, désespoir, tentatives pour le retrouver, changement de statut, etc.) ; le retour de l'anneau, ou sa perte définitive, consacrent la fin du récit. Qu'il retourne à son premier possesseur, ou qu'il passe entre les mains d'un autre, l'anneau parcourt une trajectoire de type circulaire qui est le fil même de l'intrigue, ce qu'un schéma simple permet d'illustrer ${ }^{12}$ :

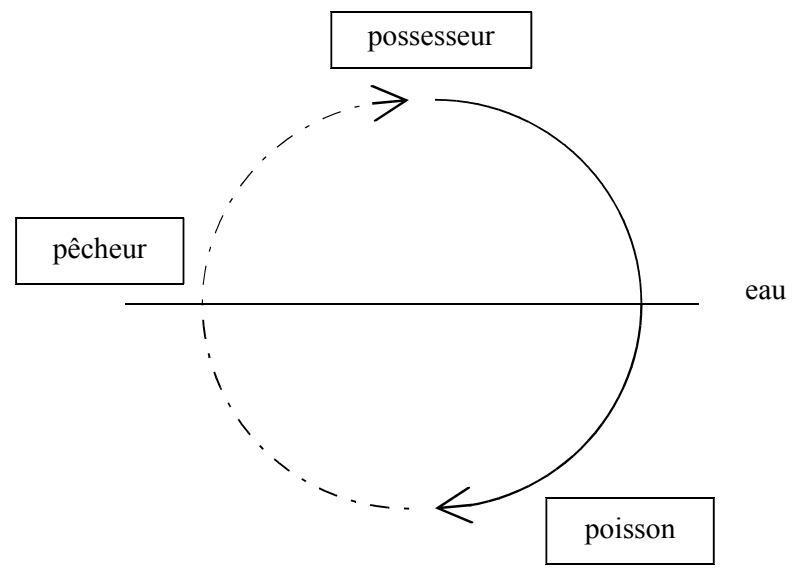

Quoiqu'ils ne puissent pas être qualifiés de 'magiques', les anneaux de ce type entretiennent un certain nombre de points communs avec les anneaux du corpus précédent. En premier lieu, ils entretiennent une relation spécifique avec leur possesseur : le statut de ce dernier, voire sa vie, sont drastiquement modifiés par la perte ou le retour

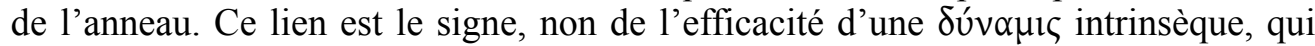
caractérisait la première catégorie d'anneaux, mais plus généralement du statut même d'objet, c'est-à-dire de 'bien possédé' $(\kappa \tau \tilde{\eta} \mu \alpha)$. L'anneau entretient une relation métonymique avec son possesseur, dont il porte la trace et qu'il représente partiellement. Le lien de possession, comme le lien d'hospitalité entre deux individus, ne définit pas deux égaux, mais deux parties indissolublement liées par une définition qui les fait se rejoindre. La dissolution du lien a pour conséquence la redéfinition radicale des deux parties. L'anneau, en tant qu'il est objet, a donc dans l'intrigue un rôle qui entre en opposition et en complémentarité avec celui de personnage ${ }^{13}$.

L'une des mises en scène les plus célèbres de cette histoire d'anneau et de propriétaire est celle du tyran Polycrate, dont il existe de nombreuses versions dans l'Antiquité, qui remontent toutes aux Histoires d'Hérodote ${ }^{14}$. Un résumé général de l'histoire

${ }^{12} C f$. Delattre (2009) 47-64.

${ }^{13}$ Certaines versions tendent d'ailleurs à faire de l'anneau un personnage animé, en le dotant d'une volonté propre : c'est le cas en particulier dans le Seigneur des anneaux de Tolkien, où l'anneau maléfique est à la fois le récipiendaire des pouvoirs de son maître, le relais de sa volonté, l'émanation de son esprit et un personnage autonome, qui a pour volonté particulière de retrouver son maître. $C f$. DelatTRE (2012).

${ }^{14} \mathrm{Si}$ l'on en croit Suétone, Néron (VI), 40, Néron lui-même s'était implicitement comparé à Polycrate au détour d'un bon mot. 
se conforme en tous points au schéma cyclique qui a été précédemment défini : Polycrate, qui dirigeait Samos d'une main de fer, se considérait lui-même comme heureux en toutes choses, mais il fut pris de peur à l'idée que cela change. Sur les conseils d'Amasis, pharaon d'Égypte et allié de Samos, il chercha à se débarrasser de l'objet qui lui était le plus précieux, afin de connaître une part de malheur. Il jeta donc à la mer un anneau qu'il affectionnait particulièrement, et se désola, pensant ne jamais le retrouver. Mais un poisson avala l'anneau, ce poisson fut pêché par un pêcheur, qui le céda au cuisinier du palais, qui le servit à Polycrate. Le tyran retrouva donc, sans qu'il y fût pour rien, l'anneau qui devait garantir son malheur présent, ainsi que la félicité. Quelques années plus tard, Polycrate paya ce bonheur en connaissant une fin ignominieuse des mains du perse Oroïtès.

Le résumé qui est présenté ici rapporte en fait deux passages distincts des Histoires d'Hérodote, un ensemble de dix-huit paragraphes, et un autre de quatre, qui est explicitement présenté comme la conséquence et la conclusion du premier ${ }^{15}$. Le résumé ne correspond donc pas exactement à la stratégie narrative d'Hérodote, à laquelle il impose une cohérence plus grande que celle qui est décelable à la lecture (ou à l'audition) du texte. Le cycle de l'anneau gagne donc en clarté, car il est directement superposé au cycle du bonheur et du malheur que connaît Polycrate, ce que l'organisation du livre III permet de déduire, mais ne met pas ouvertement en scène.

Il est malgré tout frappant de constater dans ce résumé la présence de termes qui sont ceux-là même qui organisaient le récit de Platon sur l'anneau de Gygès. Tout d'abord, la vie de Polycrate est marquée par son désir de 'pouvoir' (åpxท́) et par sa

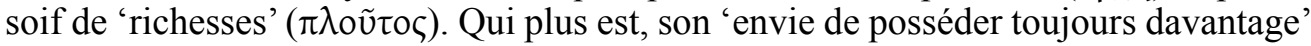

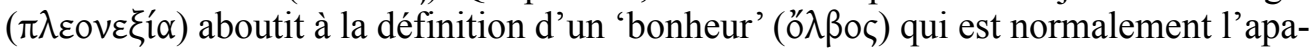
nage des dieux, et qui était aussi celui de Gygès, 'comparable aux dieux' (iøó $\theta \varepsilon \circ \varsigma$ ). Ces termes, qui régissent la vie de Polycrate dans le récit d'Hérodote, obéissent à un schéma qui est défini dans le livre I, au cours de la rencontre entre Solon et Crésus, qui organise de ce fait un pacte de lecture à l'aune duquel, du point de vue d'Hérodote, doit s'interpréter l'anecdote du tyran de Samos ${ }^{16}$.

La vie et la mort de Polycrate confirment la morale élaborée par Solon, une morale que Crésus ne comprend d'ailleurs pas tout de suite : l'être humain (óv $\theta \rho \omega \pi \circ \varsigma)$ est soumis à un cycle sans fin de bonheur et de désagrément, sans connaître jamais la 'félicité absolue' (ő $\lambda ß \varsigma \varsigma)$, qui est un privilège réservé aux dieux ${ }^{17}$. Le cycle de l'anneau exerce un triple rôle dans le récit d'Hérodote : il explique le destin de Polycrate ; il explique plus généralement le destin des hommes ; il résonne comme un écho du conseil que Crésus donne à Cyrus, une fois que lui-même a compris et assimilé les propos de Solon, à savoir qu'il existe un

${ }^{15}$ Hérodote, III, 39-56 \& 122-125. Ce travail de résumé déformant est déjà pratiqué dans l'Antiquité : cf. Libanios, Progymnasmata, II, 11, 1.

${ }^{16}$ Hérodote, I, 30-33 ; $c f$. Delattre (2009) 130-134.

${ }^{17}$ Pour les emplois du terme ő $\lambda \beta 0 \varsigma, c f$. DE HeER (1969) ; sur la stabilité de la condition humaine, cf. Lateiner (1982) et Spira (1984). Pour d'autres conceptions du bonheur dans l'Antiquité, $c f$. Jost \& SHINER (2003). 
«cycle des affaires humaines qui, dans son cours, ne permet pas que ce soient toujours les mêmes qui soient heureux» ${ }^{18}$.

\section{LE 'POUVOIR MAGIQUE' ET SES ÉQUIVALENTS}

Entre anneaux magiques et anneaux cycliques, nous venons de le voir, il existe des points de rencontre, des thématiques communes, qui rendent perméable la stricte frontière que nous avons dressée entre objets à priori dotés d'un pouvoir magique intrinsèque et objets qui n'en sont pas dotés. De quel type est donc ce pouvoir, que le grec

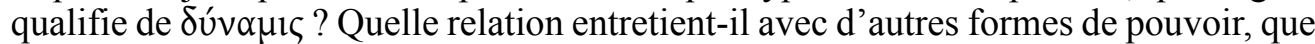

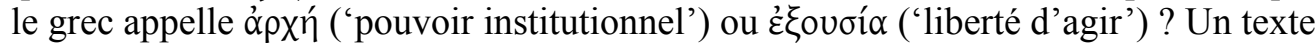
de Lucien permet d'analyser cela avec davantage de précision.

Dans une discussion animée, un personnage du nom de Timoléon explique à Lycinos quel serait son plus cher désir, à savoir posséder des anneaux magiques qui lui donneraient des superpouvoirs ${ }^{19}$. La longue énumération de ces pouvoirs désirés par Timoléon prend un tour à l'évidence ironique, qui fait du passage un divertissement sur le thème des anneaux magiques.

Chacun des superpouvoirs est défini comme la propriété magique d'un anneau qui s'exercerait sur le corps de son propriétaire. Si l'on retrouve bien le thème de l'invisibilité, en deuxième position de la liste, bien d'autres facultés physiques de Timoléon pourraient être transformées s'il trouvait les anneaux qu'il désire : il souhaite en particulier posséder un corps inaltérable ; une grande force musculaire ; la faculté de voler ; la faculté d'endormir ses ennemis ; la faculté d'ouvrir les portes ; celle d'attirer et de soumettre à son désir; d'entretenir et renouveler sa jeunesse ; d'être semblable à un

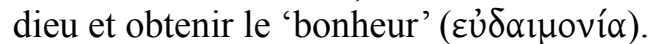

Il est notable que chacun de ces souhaits ne soit pas seulement l'expression d'un désir personnel du personnage créé par Lucien, mais traîne avec lui un ensemble de références littéraires et mythologiques, et puisse renvoyer à au moins un personnage célèbre qui a pu, temporairement ou non, posséder une de ces facultés. Le pouvoir d'éviter toute blessure fut celui de Cénée ; l'invisibilité renvoie à Gygès ; la force musculaire, à Héraclès ; le pouvoir de voler, à Icare ; la capacité d'endormir, à Hermès et sa baguette ; le pouvoir d'ouvrir les portes, à Trophonios et Agamède ; celui d'attirer et de soumettre, à Aphrodite et à sa ceinture ; la jeunesse éternelle, à Tithon ou au serpent de Glaucos. Quant à l'idée d'être semblable à un dieu et toujours heureux, elle évoque sans difficulté la figure de Crésus aussi bien que celle de Polycrate.

Le texte de Lucien confirme ainsi ce qui est apparu dans l'exposé des deux catégories d'anneau, à savoir l'existence de parallèles et de croisements entre les deux séries, pourtant définies à l'origine comme hétérogènes l'une à l'autre. Les anneaux de Lucien sont majoritairement du premier type, du fait qu'ils possèdent des pouvoirs qui ont des conséquences physiques sur les facultés corporelles de leur propriétaire,

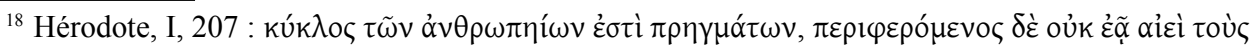

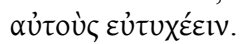

${ }^{19}$ Lucien, Navigium, 42-45. 
mais ils partagent avec les anneaux cycliques la capacité de contribuer à porter leur possesseur au comble du bonheur et de transformer son statut en le rapprochant de celui des dieux.

En définitive, la distinction établie comme une évidence à partir d'une définition stricte du 'pouvoir magique' disparaît à l'usage, et permet d'envisager une autre défi-

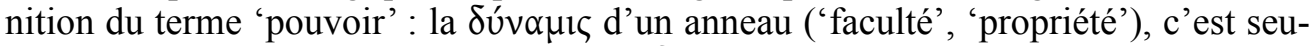
lement ce qui permet à son propriétaire de $\delta$ v́va $\sigma \theta \alpha 1$ ('avoir la faculté', 'pouvoir'). La vertu magique d'un objet a pour conséquence le pouvoir de son propriétaire, une capacité d'agir d'une manière que ses forces propres ne lui garantissent pas. Plus que de

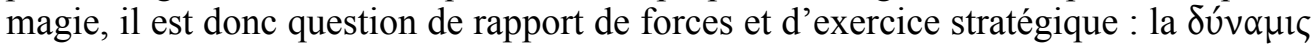
est avant tout une force agissante, une force qui s'exerce. C'est par conséquent vers le vocabulaire militaire que l'on doit se tourner d'abord pour analyser l'acte magique : de

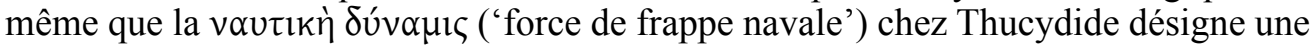
force d'intervention, une force d'impact et une capacité à se projeter sur des terrains d'opération ${ }^{20}$, de même le 'pouvoir magique' d'un anneau est avant tout un instrument

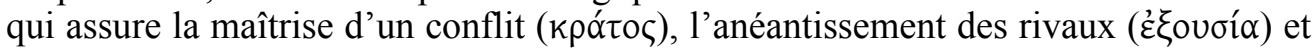
l'exercice d'un pouvoir sans contestation (’’ $\rho x \eta ́)$.

À ce titre, l'un des souhaits exprimés par Timoléon, dans le texte de Lucien, peut passer pour emblématique, car il aurait pour effet, s'il se réalisait, de transformer le pouvoir magique de l'anneau, sa propriété intrinsèque, en un véritable pouvoir s'exerçant sur les autres : Timoléon aimerait en effet par dessus tout être désirable, et il souhaiterait que ce désir s'exerce tant sur les beaux adolescents que sur les femmes, voire sur les peuples tout entiers, en bref que le monde entier soit soumis au désir, et que lui-même soit la cible absolue et la source de ce désir. Que les femmes se pendent pour lui, que les jeunes gens deviennent fous, que soit jugé heureux l'individu sur lequel se porterait un seul de ses regards, que le désespoir soit la conséquence de son mépris, bref, qu'il soit Hyacinthe, Hylas, Phaon, et plus encore, voilà l'expression de son vœu le plus cher $^{21}$.

Éros apparaît clairement dans ce texte comme une forme de pouvoir sur les autres, le moyen d'un assujettissement absolu : c'est un trait bien connu qui organise, à peu près à la même époque, l'éloge d'Amour composé par Longus dans Daphnis et Chloé,

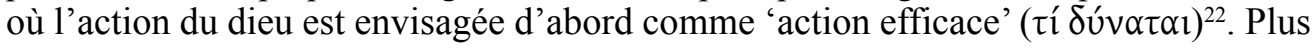

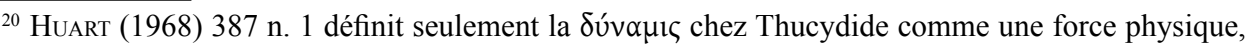
des ressources propres, une force militaire ou des possibilités d'action, qui permettent l'achèvement de

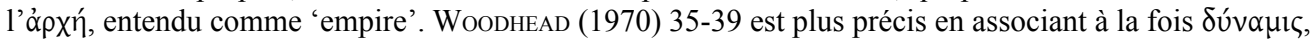

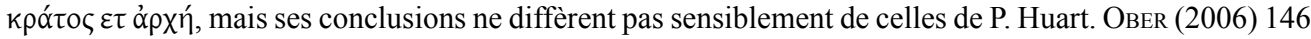

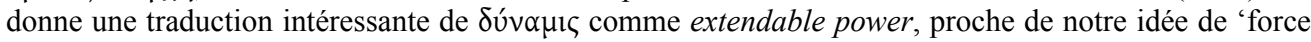
de frappe'. Sur le vocabulaire géostratégique de Thucydide et la place de la 'force de frappe navale' dans son œuvre, $c f$. DelatTRE (2001) 55-61 et 130-131.

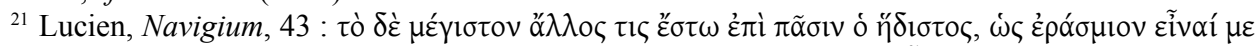

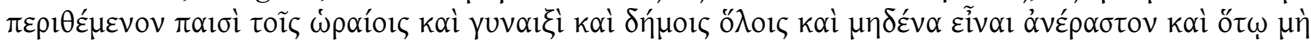

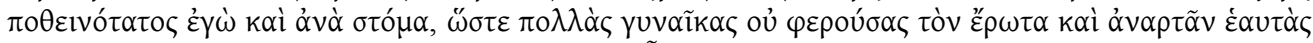

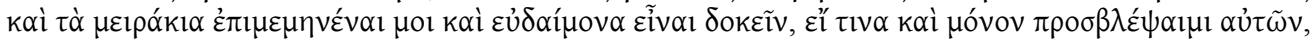

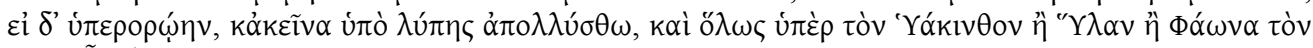

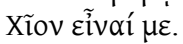

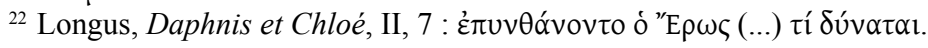




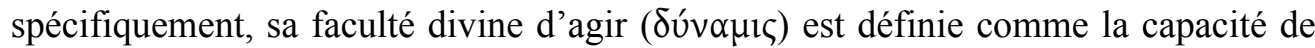

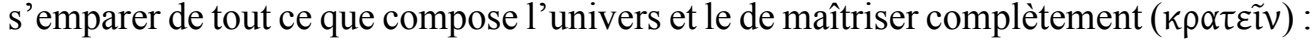

«Son pouvoir dépasse même celui de Zeus. Il domine les éléments, il domine les astres, il domine ses semblables, les dieux ; même vous n'avez pas une telle domination sur les chèvres et les moutons. ${ }^{23}$

L'éloge d'Éros chez Longus est un héritage direct des louanges adressées à Aphrodite dans l'Hymne homérique qui lui est consacré24, Aphrodite

«qui éveilla le doux désir au cœur des dieux,

et plia sous la loi les races des hommes mortels,

les oiseaux de Zeus, toutes les bêtes

que la terre nourrit en grand nombre aussi bien que la mer :

tous pensent aux travaux de Cythérée couronnée.»

(trad. J. Humbert, Belles Lettres)

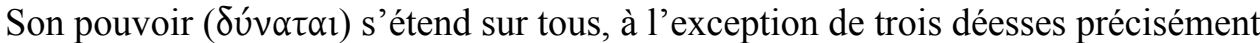
nommées dans la suite du passage, Athéna, Artémis et Hestia, et il se manifeste comme

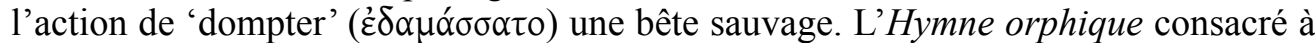
la déesse adopte les mêmes séries de métaphores pour expliciter le domaine d'action d'Aphrodite ${ }^{25}$ :

«Nocturne, qui met sous le joug, tisseuse de ruses, mère de Nécessité :

tout est de toi, tu as mis l'univers sous le joug

et tu en maîtrises les trois parts, tu engendres toutes choses,

celles qui sont dans le ciel, et sur la terre aux nombreux fruits

et dans l'abysse marin (...).

(...) c'est toi qui as mis les mortels sous le joug de nécessités sans frein,

ainsi que la foule nombreuse des bêtes grâce à des philtres qui rendent fou de désir.»

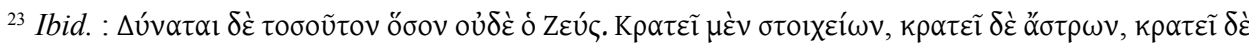

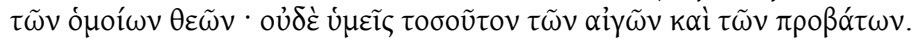

${ }^{24}$ Hymne homérique à Aphrodite (V), 2-7 :

(...)

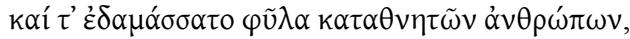

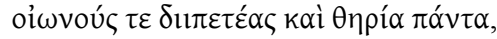

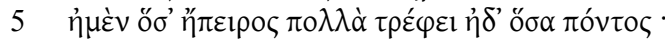

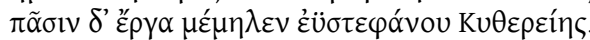

${ }^{25}$ Hymne orphique (LV), 3-7 \& 13-14 :

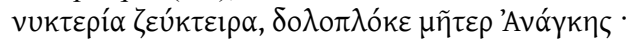

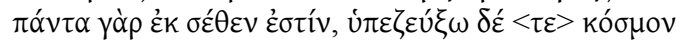

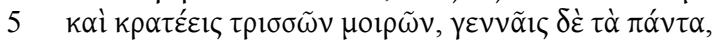

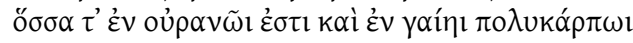

$\varepsilon \dot{\varepsilon} \vee \pi o ́ v \tau o v \tau \varepsilon \beta \nu \theta \tilde{\omega} \imath\{\tau \varepsilon\}$ (...).

(...)

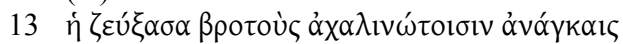

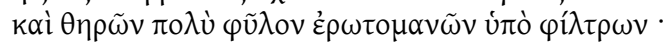


Les métaphores de l'Hymne orphique, sans grande originalité, sont fondées sur

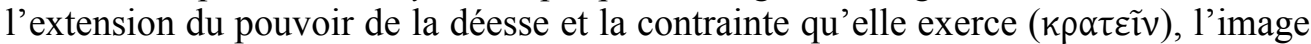

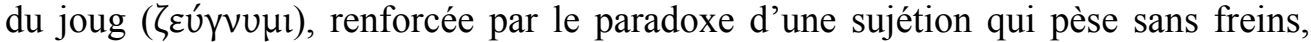

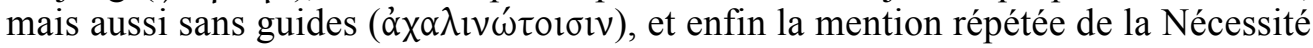

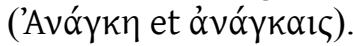

Il est inutile de fournir davantage de citations, tant la définition d'Éros et d'Aphrodite en termes de pouvoir, d'assujettissement et de mainmise est un lieu commun, et ce déjà dans la poésie lyrique de l'époque archaïque ${ }^{26}$. Plus remarquable, en revanche, est la présence, à la fois dans le texte de Lucien et dans celui de Longus, d'une équivalence implicite entre pouvoir magique, pouvoir érotique et pouvoir des plantes. Dans le Navigium de Lucien, l'interlocuteur de Timoléon, Lycinos, réplique à la longue tirade qu'il vient de subir sur les anneaux magiques par quelques remarques ironiques, voire acerbes, qu'il conclut en lui proposant un anneau, plus nécessaire que tous les autres, qui 'mettrait un terme aux bêtises' de Timoléon ( $\sigma \varepsilon \pi \alpha u ́ \sigma \varepsilon l \mu \omega \rho \alpha i ́ v o v \tau \alpha)$ et

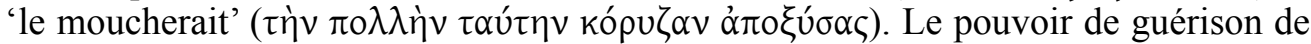
cet anneau est non seulement indiqué par le nom de la maladie qui frappe Timoléon

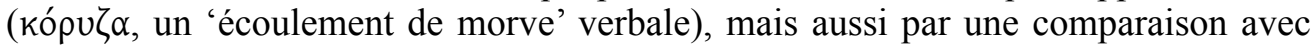
l'hellébore qui, bu en potion, serait capable d'avoir les mêmes effets ${ }^{27}$.

Or on sait que l'hellébore passait, tant dans l'Antiquité qu'au Moyen Âge ou à la Renaissance, pour avoir des propriétés purgatives si étendues qu'elles incluent la possibilité de purger les gens de leur folie ${ }^{28}$. L'anneau proposé par Lycinos à Timoléon serait donc doté d'un double pouvoir de guérison, ou plutôt d'un pouvoir qui s'exerce sur deux séries de symptômes, d'un côté un déferlement verbal, une véritable logorrhée, de l'autre le caractère déraisonnable des propos tenus. Le pouvoir magique de l'anneau est donc équivalent à celui des 'remèdes' ( $\varphi \alpha ́ \rho \mu \alpha \kappa \alpha)$, en particulier ceux que l'on tire des plantes. Et c'est bien à ces mêmes póp $\mu \alpha \kappa \alpha$ que renvoie explicitement Longus lorsqu'il évoque le fait que

«il n'existe aucun remède à l'amour, qu'on le boive, qu'on le mange, qu'on le récite dans des chansons, si ce n'est embrasser, enlacer et s'allonger sur un lit auprès d'un corps nu. $\gg^{29}$

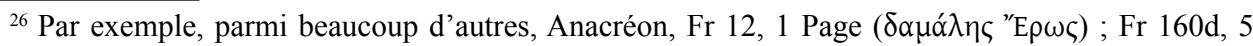

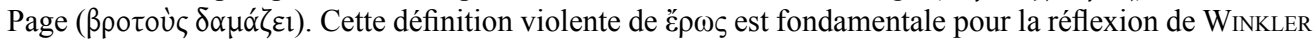
(2005) 143-196 ou celle de FARAONE (1999) 41-95, qui l'oppose à $\varphi$ i $\lambda$ í et accentue la brutalité d'un désir qui cesse d'être seulement doux-amer, comme encore chez CARSON (1986) ou chez CALAME (1996) 25-30.

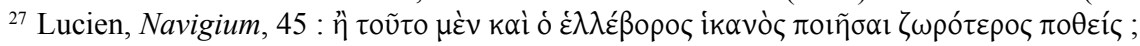

${ }^{28}$ Les textes antiques distinguent en fait un hellébore blanc (le vératre) et un hellébore noir, sans s'accorder cependant ni sur leur description, ni leurs spécificités (cf. Deroux (1976)). Il faut noter que la folie n'est qu'exceptionnellement notée dans les textes médicaux antiques comme un mal susceptible d'être guéri par l'hellébore, dont sont surtout vantées les propriétés toxiques, létales, abortives et purificatrices : $c f$. la synthèse très détaillée de STADLER (1913) et l'étude précise de GIRARD (1990).

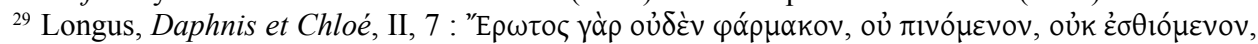

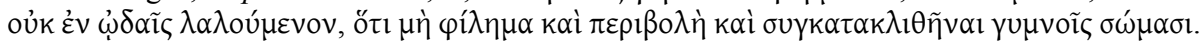


Si les remèdes à base de plantes sont efficaces, c'est qu'ils recèlent, comme les objets magiques et Éros lui-même, une $\delta u ́ v \alpha \mu ı c$. Dans le cas des plantes, il revient à la pharmacopée, entre traditions populaires des 'récolteurs de racine' ( élaboration taxinomique par Dioclès de Carystos et Théophraste, de définir à la fois la nature des plantes, leur problématique composition physique (herbe / racine / feuille / etc.), et le quelque chose qui existe en elles, qui provient de leur nature même, et qui leur donne une faculté d'agir particulière ${ }^{30}$.

Ce dernier point est très net dans un passage des Problèmes d'Aristote, qui définit la médecine et l'alimentation comme la rencontre de deux corps étrangers ${ }^{31}$ :

«la médecine est le contraire de l'aliment nourricier. Ce qui subit une coction par la nature s'assimile au corps et on l'appelle une nourriture. Au contraire ce qui n'est pas naturellement fait pour être dominé, ce qui pénètre dans les vaisseaux en y apportant le trouble par un excès de chaleur ou de froideur, voilà ce qu'est la nature d'une médecine» (trad. P. Louis).

La relation entre un corps qui ingère et un corps étranger ingéré est concue comme

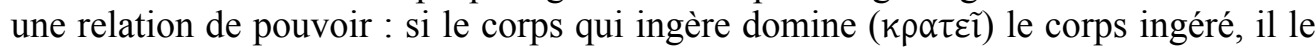
digère et se l'incorpore. Mais si le corps ingéré surpasse par ses forces propres le corps qui l'ingère, il n'y a pas incorporation, mais altération du corps qui ingère.

On voit ainsi clairement explicitée une caractéristique essentielle du pouvoir des plantes et des remèdes, qui fait que ce 'pouvoir', comme celui des objets magiques, n'est pas une image un peu vague ou une métaphore : les propriétés des uns et des autres sont proprement une $\delta u ́ v \alpha \mu \iota \zeta$, une capacité à lutter qui entre en duel pour exer-

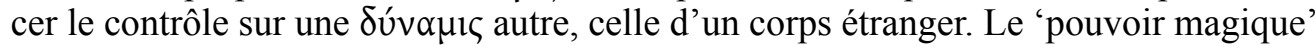
comme le 'pouvoir médical' ne prennent leur sens qu'à condition de sortir d'une définition qui les cantonne au rang de qualité intrinsèque, pour prendre en compte ce sur quoi, et surtout contre qui ils s'exercent.

Certains textes qui explicitent quels sont les pouvoirs spécifiques de telle plante ou de tel objet magique sont très clairs à ce sujet : le champ d'action est conçu comme un ring de boxe où deux adversaires s'affrontent. À titre d'exemple, il suffit de se pencher sur un texte partiellement catalogal, le De fluviis du pseudo-Plutarque, où sont énumérés entre autres des plantes et des pierres aux propriétés merveilleuses. L'auteur du texte désigne précisément à trois reprises les facultés de ces pierres et de ces plantes

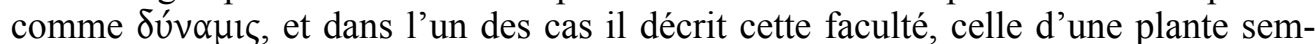
blable à la bouglosse ('langue-de-bœuf'), en identifiant ses adversaires ${ }^{32}$ :

${ }^{30} C f$. Scarborough (1991) pour une définition de la pharmacopée, ainsi que la présentation traditionnelle proposée dans DucourTHIAL (2003) 37-102.

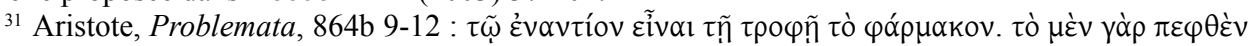

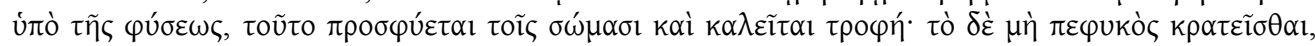

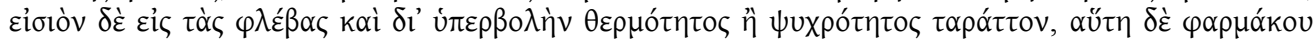

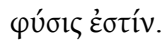

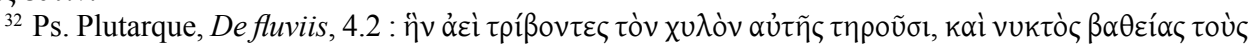

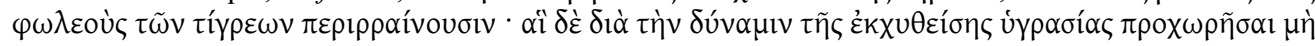


«Habituellement on la hache menu, on en conserve le suc, et on en arrose quand il fait nuit noire les abords des tanières des tigresses. Tel est le pouvoir du liquide ainsi répandu qu'elles ne peuvent plus sortir et meurent.»

\section{LE 'POUVOIR MAGIQUE' ET LE CONTRE-POUVOIR}

À la fois qualité intrinsèque dépendant de la nature et capacité à agir, le pouvoir magique d'un objet, tout comme le pouvoir thérapeutique d'une plante ou le pouvoir divin d'Éros personnifié, impliquent dans leur définition même l'existence d'un adversaire, la possibilité d'un conflit, la résolution d'un duel. De son côté, l'autre contre

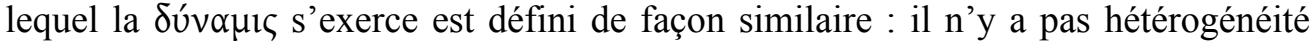
entre les participants au conflit, ils sont tous constitués de la même façon, à l'intérieur d'un espace commun qui autorise les affrontements. S'il y a pouvoir à l'intérieur d'une plante ou d'un objet, il ne s'exprime pas à partir d'un monde surnaturel contre un monde d'où tout pouvoir est absent, mais bien dans un monde qui est aussi un champ de forces diverses, d'intensités peut-être différentes, mais toutes co-présentes au sein du même monde. Seule une différence de degrés entre les différents pouvoirs qui s'expriment garantit la victoire de l'un sur l'autre.

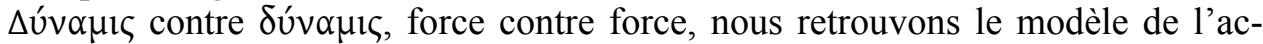
tion magique proposé par J. Favret-Saada dans Les mots, la mort, les sorts, lui-même librement inspiré des propositions d'E. de Martino ${ }^{33}$ : la magie n'y est pas conçue comme un catalogue d'actions surnaturelles, elle est représentée par ceux-là même qui prétendent la subir - ou qui éventuellement la pratiquent - comme une lutte quasiment à mort, un duel entre deux individus, définis chacun par un champ d'action et un domaine, qu'une série de schémas permettent de modéliser ${ }^{34}$.

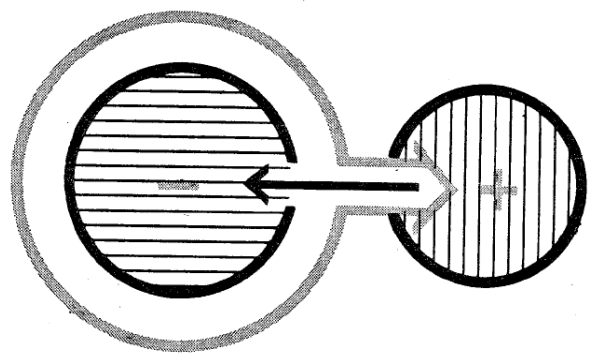

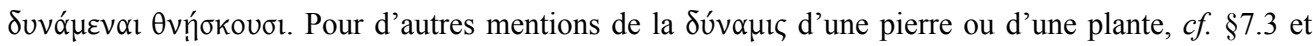
$\S 22.2$.

${ }^{33}$ Favret-SaAda (1977) ; De Martino (1948). Ce type d'analyse anthropologique s'oppose radicalement à une définition thématique de la magie, telle que celle donnée par J. Bottéro et reprise par MARTIN (2005) 12.

${ }^{34}$ L'exemple donné correspond au Schéma 4, «Une attaque de sorcellerie », FAVRET-SAADA (1977) 349, qui est explicité par un commentaire : «Ce n'est rien d'autre que le branchement de la négativité (ou de l'avidité) du sorcier sur la positivité (ou sur la fragile suffisance) d'un individu quelconque.» 
Chaque individu est animé par une force interne, qui définit son identité et circonscrit son domaine d'influence ; chacun prétend défendre son territoire ou s'approprier le territoire d'autrui. Être le propriétaire d'un objet magique, dans ce cadre, prend de nouvelles résonances : la 'propriété' (oỉkẼ̃ov) est bien ce qui caractérise l'ensemble des termes du conflit, qu'il s'agisse de l'agent ('propriétaire' de l'objet magique), de ses prétentions à être le seul maître d'un domaine qu'il estime être naturellement le sien (droit de 'propriété'), voire de l'évidence de son propre statut à ses yeux et de ce qu'on lui doit ('propriété' et convenance).

Les anneaux explicitement magiques, ceux de Lucien ou de Platon, rentrent très aisément dans ce cadre d'interprétation : un anneau magique permet d'augmenter les forces de son propriétaire ou lui accorde de nouvelles qualités pour accomplir ce qu'il n'avait, jusque là, pas le pouvoir de réaliser, c'est-à-dire pour l'emporter sur ceux qu'il ne parvenait pas à vaincre. Les anneaux non magiques, comme celui de Polycrate, sont exclus de cette définition si l'on considère seulement leur nature intrinsèque d'objet non magique. En revanche, leur nature d'objet d'énoncé permet de les y rattacher : quoiqu'ils ne soient porteurs d'aucun pouvoir, ils sont partie prenante d'un récit qui se fonde sur un duel, un conflit de pouvoirs.

Hérodote insiste à de nombreuses reprises sur ce point, qui semble correspondre dans son œuvre à une véritable Weltanschauung : ainsi l'expression remarquable 'cycle des affaires humaines', qui prétend définir la condition sans cesse changeante des hommes, entre bonheur et malheur également fugaces, ne prend son sens qu'en complément d'un énoncé plus général, donné par Crésus pour définir l'extension du pouvoir de Cyrus. Partant d'une discussion stratégique sur l'intérêt ou non de combattre les Massagètes, Crésus en vient à définir le statut général de Cyrus, celui d'un être humain au pouvoir forcément limité ( $\kappa \alpha \tau \alpha \grave{~} \delta u ́ v \alpha \mu \nu v)$ si on le compare à la puissance divine :

«si tu crois être immortel», ajoute Crésus, «et commander une armée d'immortels, te dire ma pensée ne servirait de rien ; si tu reconnais que tu es un homme, toi aussi, et que tu commandes à des hommes, laisse-moi te dire ceci d'abord : la fortune des hommes est une roue et ne laisse pas toujours les mêmes au sommet» (trad. A. Barguet) ${ }^{35}$.

La formulation hérodotéenne est bien connue, et appartient à un cadre éthique plus général qui est aussi bien celui de l'élégie de l'époque archaïque que celui de la tragédie : il existe une frontière entre les dieux et les hommes, entre immortels et mortels, entre êtres éternellement heureux et êtres éphémères. L'individu qui franchit cette frontière cosmique commet une 'agression grave' (ü $\beta \rho ı)$, un attentat au domaine

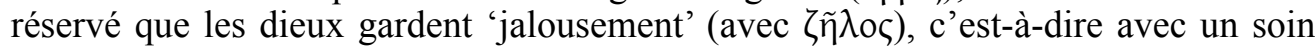
aigu de la propriétés ${ }^{6}$.

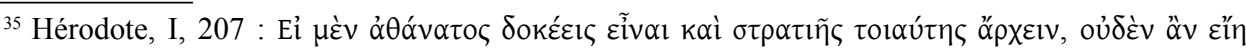

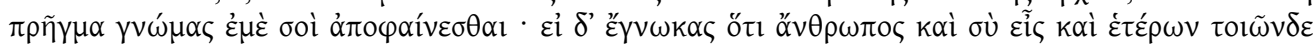

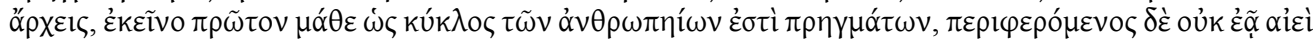

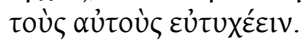

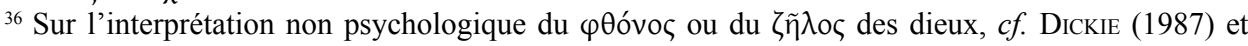
Delattre (2009) 137-139. 
Dieux et hommes, voilà les parties prenantes d'un conflit qui n'a pas lieu d'être si les êtres humains savent prendre conscience de leur humaine condition. Solon, dans son entretien avec Crésus, a formulé clairement les règles du jeu, et si Crésus peut les assener à son tour à Cyrus, c'est qu'il les a maîtrisées au prix d'un 'douloureux apprentissage ${ }^{37}$. Dans l'épisode de Polycrate, ce dernier est incapable de tirer de son aventure les leçons nécessaires, et même les conseils avisés du pharaon Amasis sont impuissants à l'éclairer sur son sort : seul le lecteur (ou auditeur), parce qu'il se rappelle l'entretien de Solon et de Crésus au livre I, et parce qu'il bénéficie des réflexions qu'Amasis garde pour lui seul, est susceptible de construire une interprétation correcte des événements :

«Au reçu de cette lettre, Amasis comprit qu'il n'était pas au pouvoir de l'homme de soustraire un homme à son destin, et qu'une fin cruelle attendait Polycrate dont le bonheur était trop complet, puisqu'il retrouvait même ce qu'il avait voulu perdre» (trad. A. Barguet) ${ }^{38}$.

Amasis, un sage, peut passer ici pour le délégué de la parole d'Hérodote ${ }^{39}$ : l'infériorité du pouvoir humain est une évidence, il est constamment limité par un 'impossible' (ódúvatov) qui implique que pour d'autres, les dieux, cela est bien 'possible' (Suvatóv).

L'anneau de Polycrate sert ainsi à la fois d'épreuve et de preuve dans un duel où Polycrate, par sa soif de richesses, son appétit de pouvoir et sa prétention au bonheur $\left(\pi \lambda \varepsilon \circ \varepsilon_{\varepsilon} \xi i ́ \alpha\right)$ affronte les dieux et l'organisation même de l'univers ${ }^{40}$.

\section{CONCLUSION}

Si nous acceptons d'abandonner une définition thématique des anneaux magiques, fondée sur la notion de surnaturel, et une définition actantielle des anneaux non magiques, fondée sur l'analyse de l'intrigue, nous aboutissons à la conclusion que peu de différences les distinguent : les uns et les autres sont partie prenante d'un même jeu de pouvoir, dans un espace défini comme un champ de forces qui s'exercent les

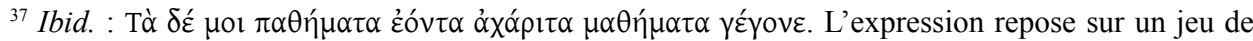

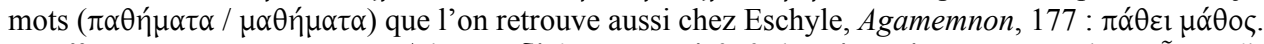

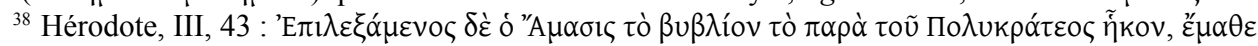

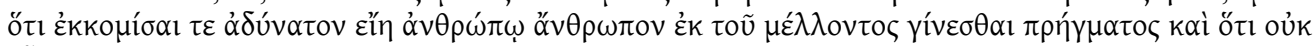

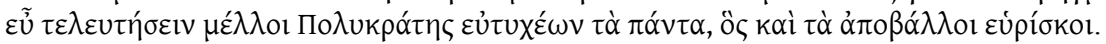

${ }^{39}$ Encore cette parole d'Hérodote n'est-elle qu'une reconstitution du lecteur : Dewald (1987) 148 a souligné que la narration d'Hérodote est faite d'éléments disjoints, comme des 'perles sur un fil' (like beads on a string) qui constituent, suivant l'expression de RenGakos (2006) 207, 'un réseau complexe d'associations qui irrigue l'ouvrage' (a complex network of associations [that] permeates the work) et qui ne sont pas soumises à un seul pacte de lecture possible. $C f$. Delattre (2009) 122.

${ }^{40}$ Pour un autre exemple d'anneau non magique impliqué dans un récit de duel, $c f$. Pausanias, I, 17, 3, et Hygin, Astronomie, II, 5, 1-4, qui résument Bacchylide, Dithyrambe XVII Snell-Maehler. Cet exemple est analysé dans Delattre (2009) 143-166. 
unes contre les autres en une lutte sans cesse renouvelée pour conquérir des territoires, garantir l'intégrité de ceux que l'on possède ou défendre ceux qui sont menacés.

C'est sans doute pour cette raison que thèmes, images et personnages ne sont pas hermétiquement enfermés à l'intérieur d'un seul ensemble, mais passent de l'un à l'autre sans difficulté : nous avons vu comment, dans le texte de Lucien, un anneau magique était susceptible de transformer quelqu'un en l'équivalent d'un dieu, thème hérodotéen par excellence. On pourra être sensible également aux variations autour de Gygès, défini par Platon comme l'heureux possesseur d'un anneau magique, mais que la poésie lyrique d'époque archaïque, puis la poésie symposiaque d'époque classique, et enfin la poésie épigrammatique d'époque romaine, ont défini comme un passionné de richesses. Archiloque a peut-être servi de prototype, en associant dans un quatrain les différents termes qui composent aussi la Weltanschauung hérodotéenne, fortune, sens de la propriété, distinction entre dieux et hommes et pouvoir absolu ${ }^{41}$ :

«je n'ai que faire des biens de Gygès, l'homme richissime, je ne suis pas la proie d'un soin jaloux, je ne suis pas à l'affût des œuvres des dieux, et je ne désire pas de grande tyrannie ; tout cela est loin de mes yeux.»

Il a été scrupuleusement suivi par l'auteur de l'un des Anacréontiques $^{42}$ :

«je n'ai que faire des biens de Gygès, le souverain de Sardes, je ne suis pas la proie de l'or, je ne suis pas jaloux des tyrans ;»

Et les mêmes expressions gouvernent encore les vers d'Alphée de Mytilène ${ }^{43}$ :

«Je n'ai pas de passion pour les labours aux épaisses moissons, ni pour la félicité dorée, telle que celle de Gygès.»

Dans cette tradition poétique, Gygès est à la fois défini par la richesse et par ses prétentions au bonheur, que les différents auteurs s'empressent de refuser, au profit soit

${ }^{41}$ Archiloque, Fr 22 Diehl = Fr 19 West apud Plutarque, Sur la tranquillité de l'âme, 470C :

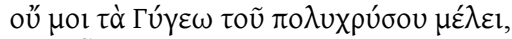

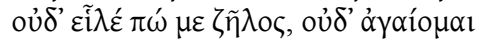

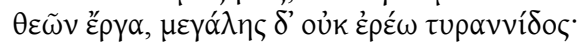

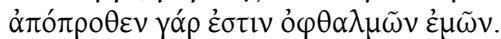

${ }^{42}$ Anacréontiques, 8 Preisendanz $=$ Anthologie Grecque, XI, 47 :

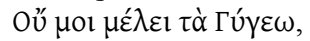

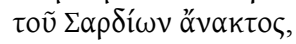

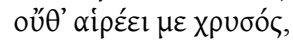

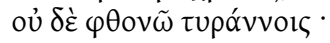

${ }^{43}$ Alphée de Mytilène, Anthologie Grecque, IX, 110 (cf. VII, 709 ; 740) :

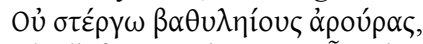

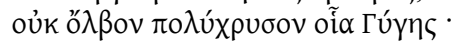


d'une vie modeste, sans plus de précision (Archiloque), soit des plaisirs du banquet,

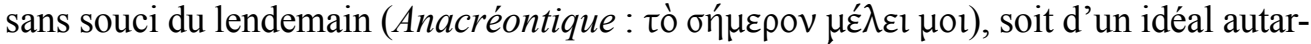
cique défini par une maxime (Alphée : $\mu \eta \theta \dot{\varepsilon} v$ ó $\gamma \alpha v$ ). Equivalent de Crésus, dont il est d'ailleurs l'ancêtre, Gygès apparaît dans ces poèmes aussi comme un alter Polycrates, c'est-à-dire comme le propriétaire par excellence d'un anneau de pouvoir, que celui-ci soit magique ou serve d'emblème au conflit dans lequel son propriétaire s'inscrit.

\section{BIBLIOGRAPHIE}

AsHerI \& alii (2007). D. Asheri, A. B. Lloyd \& A. Corcella, A Commentary on Herodotus I-IV, éd. O. Murray \& A. Moreno, Oxford, Oxford University Press, 2007.

Calame (1996). Cl. Calame, L'Éros dans la Grèce antique, coll. L'Antiquité au présent, Paris, Belin, 1996.

CARSOn (1986). A. Carson, Eros the Bittersweet. An Essay, Princeton, Princeton University Press, 1986.

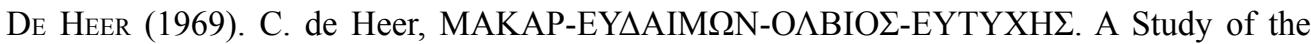
Semantic Field Denoting Happiness in Ancient Greek to the End of the $5^{\text {th }}$ Century B. C., Amsterdam, 1969.

De Martino (1948). E. de Martino, Il mondo magico. Prolegomeni a una storia del magismo, Turin, Einaudi, 1948.

Delattre (2001). Ch. Delattre, Les figures de Minos dans l'imaginaire antique. Thalassocrate, législateur, juge infernal, sous la dir. de M. Casevitz, thèse de l'Université de Paris X Nanterre, 2001.

- (2009). Ch. Delattre, Le cycle de l'anneau, de Minos à Tolkien, coll. L'Antiquité au Présent, Paris, Belin, 2009.

- (20012). Ch. Delattre, «L'anneau unique», in V. Ferré, Dictionnaire Tolkien, Paris, CNRS Éditions, 2012.

Deroux (1976). C. Deroux, «Note sur l'ellébore et le faux ellébore», Latomus, n. ${ }^{\circ} 35$, p. 875878, Bruxelles, 1976.

Dewald (1987). C. Dewald, «Narrative surface and authorial voice in Herodotus' Histories», Arethusa, n. ${ }^{\circ}$ 20, Buffalo (New York), 1987, p. 147-170.

DiCKIE (1987). M. W. Dickie, «Lo $\Phi \Theta O N O \Sigma$ degli dèi nella letteratura greca del quinto secolo avanti Cristo», Atene e Roma, n. $^{\circ}$ 32, Florence, 1987, p. 113-125.

Ducourthial (2003). G. Ducourthial, Flore magique et astrologique de l'Antiquité, préf. D. Gourevitch, coll. L'Antiquité au présent, Paris, Belin, 2003.

Faraone (1999). C. A. Faraone, Ancient Greek Love Magic, Harvard, Harvard University Press, 1999.

FaVREt-SaAda (1977). J. Favret-Saada, Les mots, la mort, les sorts, coll. Folio-Essais, Paris, Gallimard, 1977.

GiRARD (1990). M. - Chr. Girard, «L'hellébore, panacée ou placebo ?» in P. Potter, G. Maloney, J. Desautels, La maladie et les maladies dans la collection hippocratique. Actes du VI colloque international hippocratique (Québec, 28 septembre - 3 octobre 1987), Québec, Éditions du Sphinx, 1990, p. 393-405. 
HuART (1968). P. Huart, Le vocabulaire de l'analyse psychologique dans l'œuvre de Thucydide, coll. Études et commentaires, n. ${ }^{\circ}$ 69, Paris, Klincksieck, 1968.

Jost \& Shiner (2003). L. J. Jost \& R. A. Shiner (éds.), Eudaimonia and Well-Being : Ancient and Modern Conceptions, Kelowna (BC), Academic Printing and Publishing, 2003.

LATEINER (1982). D. Lateiner, «A Note on the Perils of Prosperity in Herodotus», Rheinisches Museum, n. ${ }^{\circ}$ 125, Francfort, 1982, p. 97-101.

MARTIN (2005). M. Martin, Magie et magiciens dans le monde gréco-romain, coll. Hespérides, Paris, Errance, 2005.

OBer (2006). J. Ober, «Thucydides and the Invention of Political Science», in A. Rengakos \& A. Tsakmakis (éds.), Brill's Companion to Thucydides, Leyde, Brill, 2006, p. 131-160.

Rengakos (2006). A. Rengakos, «Homer and the historians : the influence of epic narrative technique on Herodotus and Thucydides», in Fr. Montanari \& A. Rengakos (éds.), La poésie épique grecque : métamorphoses d'un genre littéraire. Vandouvres-Genève, 22-26 août 2005, coll. Entretiens sur 1'Antiquité classique, n. ${ }^{\circ}$ 52, Vandœuvres-Genève, Fondation Hardt, 2006, p. 182-209.

Scarborough (1991). J. Scarborough, «The Pharmacology of Sacred Plants, Herbs, and Roots», in C. A. Faraone \& D. Obbink (éds.), Magika Hiera. Ancient Greek Magic and Religion, Oxford, Oxford University Press, 1991, p. 139-174.

SPIRA (1984). A. Spira, «'Stabilität' und 'Instabilität' in der Ethik der Griechen», Zeitschrift für Religion und Geistesgeschichte, n. ${ }^{\circ} 36,1984$, p. 115-130.

Stadler (1913)- H. Stadler, s. v. Helleboros, éd. Pauly, G. Wissowa, W. Kroll, Realencyclopädie der classischen Altertumswissenschaft (RE), t. VIII, col. 163-170, Stuttgart, Metzler, 1913.

Winkler (2005). J. J. Winkler, Désir et contraintes en Grèce ancienne, trad. S. Boehringer \& N. Picard, coll. les grands classiques de l'érotologie moderne, Paris, EPEL, 2005.

Woodhead (1970). A. G. Woodhead, Thucydides and the nature of power, Martin Classical Lectures, n. ${ }^{\circ}$ 24, Cambridge (Ma), Harvard University Press, 1970. 